however, that they did not report effects of pyridoxamine on the root cause of vaso-occlusion, i.e., the sickle red blood cells (RBC). Especially at this time, when we know clearly that sickle RBC are deficient in anti-oxidant capacity ${ }_{15}^{15}$ and that oxidative pathways in RBC promote vasoocclusion, ${ }^{13}$ understanding the effects of anti-oxidant therapies on sickle RBC biology, the root cause of SCD, should be prioritized.

\section{References}

1. Telen MJ, Malik P, Vercellotti GM. Therapeutic strategies for sickle cell disease: towards a multi-agent approach. Nat Rev Drug Discov. 2019;18(2):139-158.

2. Li J, Jeong SY, Xiong B, et al. Repurposing pyridoxamine for therapeutic intervention of intravascular cell-cell interactions in mouse models of sickle cell disease. Haematologica. 2020;105(10):2407-2419.

3. Chen JL, Francis J. Pyridoxamine, advanced glycation inhibition, and diabetic nephropathy. J Am Soc Nephrol. 2012;23(1):6-8.

4. Lewis EJ, Greene T, Spitalewiz S, et al. Pyridorin in type 2 diabetic nephropathy. J Am Soc Nephrol. 2012;23(1):131-136.

5. Raval AD, Thakker D, Rangoonwala AN, Gor D, Walia R. Vitamin B and its derivatives for diabetic kidney disease. Cochrane Database Syst Rev. 2015;1:CD009403.
6. Nur E, Brandjes DP, Schnog JJ, et al. Plasma levels of advanced glycation end products are associated with haemolysis-related organ complications in sickle cell patients. Br J Haematol. 2010;151(1):62-69.

7. Niihara Y, Smith WR, Stark CW. A phase 3 trial of L-glutamine in sickle cell disease. N Engl J Med. 2018;379(19):1880.

8. Vichinsky E, Hoppe CC, Ataga KI, et al. A phase 3 randomized trial of voxelotor in sickle cell disease. N Engl J Med. 2019;381(6):509-519

9. Ataga KI, Kutlar A, Kanter J, et al. Crizanlizumab for the prevention of pain crises in sickle cell disease. N Engl J Med. 2017;376(5):429-439.

10. Belcher JD, Chen C, Nguyen J, et al. Control of oxidative stress and inflammation in sickle cell disease with the Nrf2 activator dimethyl fumarate. Antioxid Redox Signal. 2017;26(14):748-762.

11. Morris CR, Suh JH, Hagar W, et al. Erythrocyte glutamine depletion, altered redox environment, and pulmonary hypertension in sickle cell disease. Blood. 2008;111(1):402-410.

12. Nur E, Biemond BJ, Otten HM, Brandjes DP, Schnog JJ. Oxidative stress in sickle cell disease; pathophysiology and potential implications for disease management. Am J Hematol. 2011;86(6):484-489.

13. Thamilarasan M, Estupinan R, Batinic-Haberle I, Zennadi R. Mn porphyrins as a novel treatment targeting sickle cell NOXs to reverse and prevent acute vaso-occlusion in vivo. Blood Adv. 2020;4(11): 23722386

14. Ramis R, Ortega-Castro J, Caballero C, et al. How does pyridoxamine inhibit the formation of advanced glycation end products? The role of its primary antioxidant activity. Antioxidants (Basel). 2019;8(9):344.

15. Sangokoya C, Telen MJ, Chi JT. microRNA miR-144 modulates oxidative stress tolerance and associates with anemia severity in sickle cell disease. Blood. 2010;116(20):4338-4348.

\title{
ARQ531: the therapy that targets multiple pathways in acute myeloid leukemia
}

\author{
Charlotte Hellmich, ${ }^{1,2}$ Kristian Bowles ${ }^{1,2}$ and Stuart Rushworth ${ }^{1}$
}

${ }^{1}$ Norwich Medical School, University of East Anglia, Norwich Research Park, Norwich and 'Department of Haematology, Norfolk and Norwich University Hospitals NHS Trust, Norwich, UK

E-mail: STUART RUSHWORTH - s.rushworth@uea.ac.uk

doi:10.3324/haematol.2020.257022

S o far this century we have witnessed the introduction of a number of targeted therapies, developed through rational drug design, which have changed cancer treatment and resulted in improved outcomes for many patients, including those with a spectrum of chronic lymphoid and myeloid malignancies. ${ }^{1,2}$ However, despite improved understanding of the biology of acute myeloid leukemia (AML), similar scale benefits by targeting kinases and other intracellular and surface proteins have yet to be realized, and the prognosis for patients with AML remains poor. Moreover, cytotoxic drugs and therapies developed in the last century currently remain the backbone of AML treatment, and as AML primarily affects the elderly, many of whom are therefore frail with multiple co-morbidities, the clinical application of such curative therapies is somewhat limited. ${ }^{3}$ Furthermore, even in those fit enough for intensive chemotherapy, both relapse and treatment resistance are common, due to the aggressive nature of the disease. The search therefore continues for biology-driven targeted treatments for patients with AML which can be delivered to all, and at the same time increase remission rates, reduce relapses and prevent treatment resistance. The expectation is that these therapies will come from advances in the understanding of the biology of AML.
Tyrosine kinases are known to play a role in the tumorigenesis of both solid tumors and hematological malignancies and they therefore present a potential treatment target. ${ }^{4}$ In particular, Bruton tyrosine kinase (BTK) inhibitors have been shown to be effective in the treatment of a number of hematologic malignancies including chronic lymphocytic leukemia and lymphomas. ${ }^{5-7}$ BTK is a non-receptor tyrosine kinase with an important role in both normal and malignant hematopoiesis. ${ }^{8}$ Its expression and phosphorylation are high in AML and BTK inhibition with ibrutinib has been shown to have an anti-leukemic effect.' Moreover, many other tyrosine kinases have been shown to be activated in AML and hematologic malignancies including SYK, FLT3, MAPK, PI3K and AKT..$^{10,11}$ This knowledge supports further exploration of tyrosine kinase inibitors in the treatment of AML.

In this issue of Haematologica, Soncini et al. explore the potential role of ARO531, a reversible small molecule inhibitor of BTK and a number of other kinases, in the management of $\mathrm{AML}^{12}$ (Figure 1). BTK was shown to be constitutively active in a range of different subtypes of AML, suggesting that targeting it could have a broad clinical application in all patients with AML. ARQ531 was observed to have dose-dependent anti-leukemic activity by inducing apoptosis in both AML cell lines and primary AML cells. 


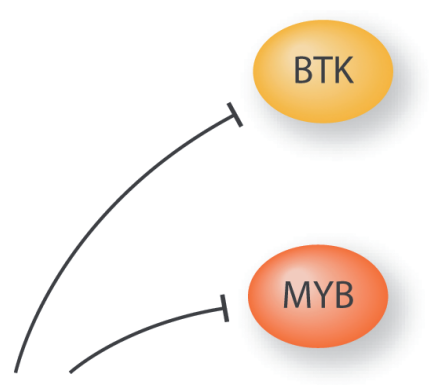

ARQ531
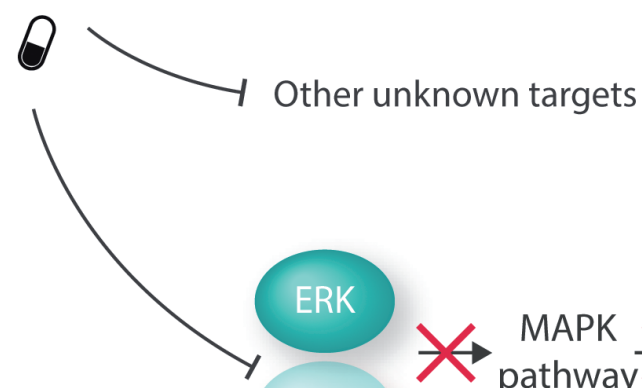

Other unknown targets

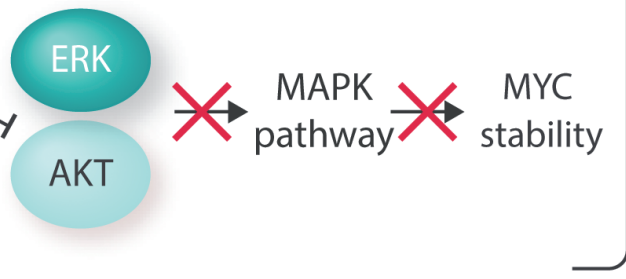

Impaired AML engraftment

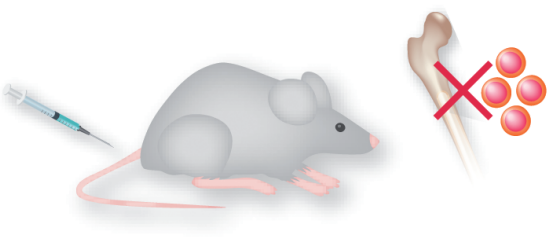

Reduced tumor burden

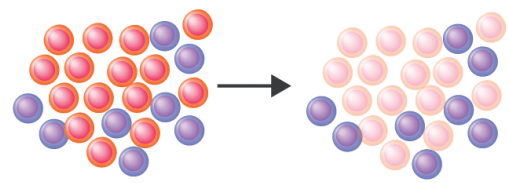

Improved animal survival

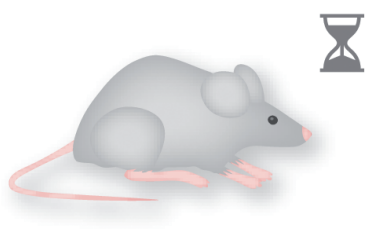

Figure 1. Schematic representation of the mechanism of action of ARQ531 in acute myeloid leukemia. BTK: Bruton tyrosine kinase; ERK: extracellular-signal-related kinase; MAPK: mitogen-activated protein kinase; AML: acute myeloid leukemia.

The effect of ARQ531 was superior to that of treatment with ibrutinib and this was likely due to the broader mode of action of ARO531. While ARQ531 has anti-BTK activity its action does not appear to be dependent on BTK and it was shown to inhibit a number of other oncogenic pathways as well. Thus, ARO531 treatment reduced cell viability even after knock-down of BTK in AML cells.

We know that AML is heavily reliant on its tumor microenvironment ${ }^{13}$ and disrupting this relationship is a crucial step in improving AML treatments and therefore outcomes for patients. When considering effectiveness of new treatments, we must therefore assess whether they are able to target leukemic cells within this supportive environment. Soncini et al. were able to demonstrate that the antileukemic activity of ARQ531 was preserved when AML cells were co-cultured with mesenchymal stromal cells. Moreover, the viability of the mesenchymal stromal cells as well as that of other non-malignant cells, including hematopoietic stem cells, was not affected by treatment with ARO531. This is important when considering the potential toxicity of a drug as there is a need to protect nonmalignant cells in order to prevent side effects and reduce treatment-related morbidity and mortality.

A number of oncogenic pathways have been shown to drive leukemogenisis, disease progression and treatment resistance. In addition to upregulation of BTK, these include activation of the mitogen-activated protein kinase (MAPK) pathway and dysregulation of the hematopoietic transcriptional factor MYB, which have been implicated in the pathogensis of AML. ${ }^{14,15}$ The mode of action of ARQ531 is complex and genetic analysis revealed that it inhibits a number of different oncogenic pathways in AML. The data published in this issue show that ARQ531 disrupts the oncogenic MAPK pathway by inhibiting ERK and AKT activation as well as downregulating MYC. Furthermore, ARQ531 was shown to deregulate and promote degradation of the oncogenic driver MYB. Interestingly the action on all of these pathways, including BTK, MAPK and MYB, as well, potentially, as others not yet identified, appears to have a cumulative effect on cell viability. Inhibition of each pathway individually is less effective in reducing cell viability than using ARQ531, suggesting that additional pathways are important in this drug's mode of action. It is clear that leukemogenesis relies on the dysregulation of multiple oncogenic pathways and therefore targeting only one of these is not sufficient to achieve cell death and reduce tumor growth. A drug that has the ability to target a number of these pathways does, therefore, have greater potential to effectively eliminate malignant cells and at the same time has little or no lasting effect on non-malignant cells.

To demonstrate this Soncini et al. designed an in vivo experiment using a patient-derived AML mouse model. Following engraftment of immunocompromised mice with primary AML cells they demonstrated that treatment with ARO531 impaired AML engraftment, reduced tumor burden and improved the animals' survival. Furthermore, there were no signs of toxicity, suggesting that ARQ531 could be an effective and well-tolerated treatment for AML in the 
future. However, a note of caution is that many potential therapies have been shown to have in vivo efficacy in AML, but when tested clinically have had little or no effect on this disease.

In conclusion, by targeting a number of different oncogenic pathways, in vitro and in vivo treatment with ARO531 results in reduced AML cell viability, reduced tumor growth and improved survival of animals. The research by Soncini et al. suggests that a multi-targeted inhibitor such as ARO531 is required to impair AML survival effectively; since this drug does not rely specifically on high expression of BTK or other tyrosine kinases it could be widely applicable to different subtypes of AML.

\section{References}

1. Longo DL. Imatinib changed everything. $\mathrm{N}$ Engl J Med. 2017;376(10):982-983.

2. Yosifov DY, Wolf C, Stilgenbauer S, Mertens D. From biology to therapy: the CLL success story. HemaSphere. 2019;3(2):e175.

3. Juliusson G, Antunovic P, Derolf A, et al. Age and acute myeloid leukemia: real world data on decision to treat and outcomes from the Swedish Acute Leukemia Registry. Blood. 2009;113(18):4179-4187.

4. Hanahan D, Weinberg RA. Hallmarks of cancer: the next generation. Cell. 2011;144(5):646-674.

5. Byrd JC, Furman RR, Coutre SE, et al. Targeting BTK with ibrutinib in relapsed chronic lymphocytic leukemia. $N$ Engl J Med.
2013;369(1):32-42.

6. Wang ML, Rule S, Martin P, et al. Targeting BTK with ibrutinib in relapsed or refractory mantle-cell lymphoma. N Engl J Med. 2013;369(6):507-516.

7. Treon SP, Tripsas CK, Meid K, et al. Ibrutinib in previously treated Waldenström's macroglobulinemia. N Engl J Med. 2015;372(15): 1430-1440

8. Rushworth SA, Pillinger G, Abdul-Aziz A, et al. Activity of Bruton's tyrosine-kinase inhibitor ibrutinib in patients with CD117-positive acute myeloid leukaemia: a mechanistic study using patient-derived blast cells. Lancet Haematol. 2015;2(5):e204-211.

9. Rushworth SA, Murray MY, Zaitseva L, Bowles KM, MacEwan DJ. Identification of Bruton's tyrosine kinase as a therapeutic target in acute myeloid leukemia. Blood. 2014;123(8):1229-1238.

10. Chalandon Y, Schwaller J. Targeting mutated protein tyrosine kinases and their signaling pathways in hematologic malignancies. Haematologica. 2005;90(7):949-968.

11. Fernandez S, Desplat V, Villacreces A, et al. Targeting tyrosine kinases in acute myeloid leukemia: why, who and how? Int J Mol Sci. 2019;20(14).

12. Soncini $\mathrm{D}$, Orecchioni $\mathrm{S}$, Ruberti $\mathrm{S}$, et al. The new small molecule tyrosine-kinase inhibitor ARQ531 targets acute myeloid leukemia cells by disrupting multiple tumor-addicted programs. Haematologica. 2020; 105(10):2420-2431.

13. Shafat MS, Gnaneswaran B, Bowles KM, Rushworth SA. The bone marrow microenvironment - home of the leukemic blasts. Blood Rev. 2017;31(5):277-286.

14. Milella M, Kornblau SM, Estrov Z, et al. Therapeutic targeting of the MEK/MAPK signal transduction module in acute myeloid leukemia. J Clin Invest. 2001;108(6):851-859.

15. Frech M, Teichler S, Feld C, et al. MYB induces the expression of the oncogenic corepressor SKI in acute myeloid leukemia. Oncotarget. 2018;9(32):22423-22435.

\section{A step ahead toward precision medicine for chronic lymphocytic leukemia}

\section{Andrea Patriarca and Gianluca Gaidano}

Division of Hematology, Department of Translational Medicine, University of Eastern Piedmont and Maggiore Charity Hospital, Novara, Italy

E-mail: GIANLUCA GAIDANO - gianluca.gaidano@uniupo.it

doi:10.3324/haematol.2020.257048

1 he concept of precision medicine applied to human tumors implies the personalized tailoring of clinical management and treatment choices according to the status of an array of molecular biomarkers, in conjunction with other patient features. ${ }^{1}$ In chronic lymphocytic leukemia (CLL), the extensive body of genetic data that have been accumulated in recent years has led to the identification of many new molecular biomarkers with prognostic value. However, only a few of these serve the role of true predictors for choosing the most appropriate treatment for any given patient. ${ }^{1,2}$ The active search for molecular predictors in CLL is becoming increasingly more important in the current therapeutic landscape of the disease, that ranges from chemo-immunotherapy with both old and newer monoclonal antibodies (mAb) to chemo-free options based on B-cell receptor (BCR) inhibitors, targeting either Bruton tyrosine kinase or phosphatidyilinositol-3-kinase, and BCL2 inhibitors. ${ }^{3,4}$

In this issue of Haematologica, Tausch et al. have analyzed the prognostic and, more importantly, the predictive role of a panel of gene mutations in the randomized, phase III COMPLEMENT1 trial comparing chlorambucil with ofatumumab-chlorambucil in treatment-naive CLL patients not eligible for intensive therapy because of age or comorbidities. ${ }^{5}$ The COMPLEMENT 1 trial had documented that addition of the type 1 anti-CD20 mAb ofatumumab to chlorambucil leads to clinically significant improvement in progression-free survival (PFS) (22.4 months in the arm treated with ofatumumab chlorambucil vs. 13.1 months in the arm treated with single agent chlorambucil), with a manageable side effect profile. ${ }^{6}$ But whether ofatumumab provided an advantage to all molecular subgroups of CLL remains unexplored. Remarkably, in the genetic analysis performed by Tausch et al., mutations of NOTCH1 were seen to predict weak benefit from the addition of ofatumumab to the chlorambucil backbone. ${ }^{5}$ The NOTCH1 signaling pathway is a key feature in CLL growth and survival, and is deregulated by mutations in a sizable fraction of $\mathrm{CLL}^{7}$ (Figure 1). NOTCH1 mutations in CLL may target either the autoregulatory PEST domain, or the non-coding 3'untranslated region (3'-UTR) sequence. ' In the context of 